\title{
Magnetic Sphincter Augmentation for Gastroesophageal Reflux at 5 Years: Final Results of a Pilot Study Show Long-Term Acid Reduction and Symptom Improvement
}

\author{
Greta Saino, MD, ${ }^{1, *}$ Luigi Bonavina, MD, ${ }^{1, *}$ John C. Lipham, MD, ${ }^{2}$ \\ Daniel Dunn, MD, ${ }^{3}$ and Robert A. Ganz, MD ${ }^{4}$
}

\begin{abstract}
Background: As previously reported, the magnetic sphincter augmentation device (MSAD) preserves gastric anatomy and results in less severe side effects than traditional antireflux surgery. The final 5-year results of a pilot study are reported here.

Patients and Methods: A prospective, multicenter study evaluated safety and efficacy of the MSAD for 5 years. Prior to MSAD placement, patients had abnormal esophageal acid and symptoms poorly controlled by proton pump inhibitors (PPIs). Patients served as their own control, which allowed comparison between baseline and postoperative measurements to determine individual treatment effect. At 5 years, gastroesophageal reflux disease (GERD)-Health Related Quality of Life (HRQL) questionnaire score, esophageal pH, PPI use, and complications were evaluated.

Results: Between February 2007 and October 2008, 44 patients (26 males) had an MSAD implanted by laparoscopy, and 33 patients were followed up at 5 years. Mean total percentage of time with $\mathrm{pH}<4$ was $11.9 \%$ at baseline and $4.6 \%$ at 5 years $(P<.001)$, with $85 \%$ of patients achieving $\mathrm{pH}$ normalization or at least a $50 \%$ reduction. Mean total GERD-HRQL score improved significantly from 25.7 to $2.9(P<.001)$ when comparing baseline and 5 years, and $93.9 \%$ of patients had at least a $50 \%$ reduction in total score compared with baseline. Complete discontinuation of PPIs was achieved by $87.8 \%$ of patients. No complications occurred in the long term, including no device erosions or migrations at any point.

Conclusions: Based on long-term reduction in esophageal acid, symptom improvement, and no late complications, this study shows the relative safety and efficacy of magnetic sphincter augmentation for GERD.
\end{abstract}

\section{Introduction}

G ASTROESOPHAGEAL REFLUX DISEASE (GERD) is characterized by the retrograde movement of gastric contents through the lower esophageal sphincter (LES) into the esophagus, resulting in bothersome symptoms and/or damage to esophageal tissue. ${ }^{1}$ Patients with GERD are routinely treated with proton pump inhibitors (PPIs), which aim to alleviate symptoms related to acid reflux by suppressing normal acid production in the stomach, effectively making the gastric refluxate less acidic. ${ }^{2}$ Although PPIs are effective in managing symptoms of heartburn and healing esophagitis, they are unable to address an incompetent LES and therefore fail to prevent the occurrence of pathologic reflux. It is therefore not surprising that patients experience regurgitation and non-acidic-related symptoms of GERD despite PPI use. ${ }^{3-5}$

\footnotetext{
${ }^{1}$ Division of General Surgery, IRCCS Policlinico San Donato, University of Milan Medical School, Milan, Italy.

${ }^{2}$ Department of Surgery, Keck School of Medicine, University of Southern California, Los Angeles, California.

${ }^{3}$ Division of Surgery, Abbot Northwestern Hospital, Minneapolis, Minnesota.

${ }^{4}$ Minnesota Gastroenterology PA, Plymouth, Minnesota.

*The first two authors contributed equally to this study and share co-first authorship.

This study is registered with ClinicalTrials.gov with clinical trial registration number NCT0105870.
}

(C) The Author(s) 2015; Published by Mary Ann Liebert, Inc. This Open Access article is distributed under the terms of the Creative Commons Attribution Noncommercial License (http://creativecommons.org/licenses/by-nc/4.0/) which permits any noncommercial use, distribution, and reproduction in any medium, provided the original author(s) and the source are credited. 
The shortcomings of acid-suppression therapy are addressed by antireflux surgery, traditionally Nissen fundoplication, and treatment is directed at restoring normal function of the antireflux barrier by reconstructing a defective LES. ${ }^{6}$ This approach, although effective at controlling reflux, requires significant anatomical disruption in order to mobilize the gastric fundus and wrap it around the esophagus. ${ }^{7,8}$

The magnetic sphincter augmentation device (MSAD) $\left(\right.$ LINX $^{\circledR}$ Reflux Management System; Torax Medical, Shoreview, MN) was developed as a less disruptive surgical option for the treatment of GERD. ${ }^{9}$ The MSAD is a small implant composed of interlinked titanium beads with magnetic cores. The magnetic attraction between the beads augments the existing LES barrier function to prevent reflux. The device is implanted using a standard minimally invasive laparoscopic procedure. Interim reports of the initial cohort of patients implanted with MSAD have been published. ${ }^{10-12}$ We now provide here a final report of the 5 -year clinical results.

\section{Subjects and Methods}

The study was a prospective, multicenter, single-arm study, where patients served as their own control, allowing comparison of baseline and postoperative measurements after MSAD placement to determine treatment effect. The study enrolled 44 patients at four clinical sites (two in the United States and two in Europe). The study protocol was approved by the institutional review board or ethics committee at each clinical site, and written informed consent was obtained from all participants.

\section{Study patients}

All enrolled patients had abnormal esophageal $\mathrm{pH}$ as measured by ambulatory esophageal $\mathrm{pH}$ monitoring, exhibited typical GERD symptoms, had been taking daily PPIs, and were between 18 and 75 years of age. Patients were excluded if they had a large hernia $(>3 \mathrm{~cm})$, esophagitis of Grade B or higher (Los Angeles Classification), a body mass index of $>35 \mathrm{~kg} / \mathrm{m}^{2}$, Barrett's esophagus, motility disorders, or gross esophageal anatomic abnormalities. Patients with a known allergy to titanium, stainless steel, nickel, or ferrous materials were contraindicated for the MSAD and therefore were not eligible for the study.

\section{Preoperative assessment}

Preoperative assessments prior to MSAD have been previously described. ${ }^{10,11}$ In brief, patients were evaluated before surgery with a symptom questionnaire, esophageal $\mathrm{pH}$ monitoring, esophageal manometry, upper gastrointestinal endoscopy, and barium swallow. Determination of hernia size for eligibility was based on upper gastrointestinal endoscopy. The length of the hernia, if present, was measured as the distance between the gastroesophageal junction, defined by the proximal limit of the gastric folds, and the crural impression.

\section{Study device}

The study device, the MSAD, was implanted with standard laparoscopic technique and was sized to fit around the external diameter of the resting esophagus, without compression.
Details about the implant procedure have been previously described. ${ }^{10-12}$ The MSAD provides magnetic sphincter augmentation, which means the circumferential magnetic resistance provided by the device helps prevent abnormal opening of the LES, thus preventing pathologic reflux. The device consists of a series of titanium beads with magnetic cores that are connected with independent titanium wires to form an annular shape that may open and close. The beads are connected in a fashion that allows the device to expand to accommodate increased intraluminal forces such as those associated with swallowing food or with belching and vomiting.

\section{Assessments and follow-up}

The study was designed to follow all patients for at least 1 year after LINX implant. After the first year, all but one clinical site continued to follow patients annually through 5 years for GERD-Health Related Quality of Life (GERDHRQL) score, PPI use, and complications. One site elected to also perform $\mathrm{pH}$ monitoring annually through 5 years for willing patients. Clinical outcomes measured at 5 years included the GERD-HRQL score, esophageal pH measurements, PPI use, abdominal/chest x-ray, and complications.

The x-ray at follow-up was compared with the postoperative $\mathrm{x}$-ray to confirm placement of the device at the implant site.

The GERD-HRQL and $\mathrm{pH}$ monitoring were completed with PPIs discontinued for at least 10 days at baseline and at all follow-up visits. The GERD-HRQL is a self-assessed, disease-specific questionnaire designed to measure GERD symptom severity on quality of life. ${ }^{13}$ It consists of 10 questions relating to severity of heartburn symptoms while lying down, standing up, after meals, after a change in diet, and while sleeping and to severity of symptoms related to GERD, including dysphagia, odynophagia, bloating, and effect of medications. The total GERD-HRQL score represents a summation of each of the 10 items. The best possible score is 0 (i.e., asymptomatic in each item), and the worst possible scores is 50 (incapacitated in each item). The questionnaire also measured satisfaction with present condition. Treatment was considered a success if at least a $50 \%$ reduction in total GERD-HRQL score was achieved when comparing scores at follow-up and baseline. Esophageal $\mathrm{pH}$ monitoring was completed at baseline to objectively confirm the diagnosis of GERD and then repeated after MSAD placement. All patients were required to complete $\mathrm{pH}$ testing at the 1-year follow-up per protocol. Thereafter, one site continued to evaluate $\mathrm{pH}$ on an annual basis through 5-year follow-up. Measurements collected from esophageal $\mathrm{pH}$ testing included the DeMeester score and its individual components.

PPI use at follow-up was reported as complete discontinuation of PPIs or at least a 50\% reduction in dose of PPI use compared with baseline.

Adverse events were tracked throughout the duration of the clinical study, starting at the time of implant procedure until completion of the study.

\section{Statistical analysis}

Patients served as their own control, and postoperative data were compared with preoperative data to evaluate treatment effect using the two-tailed, paired Student's $t$ test. Mean and 


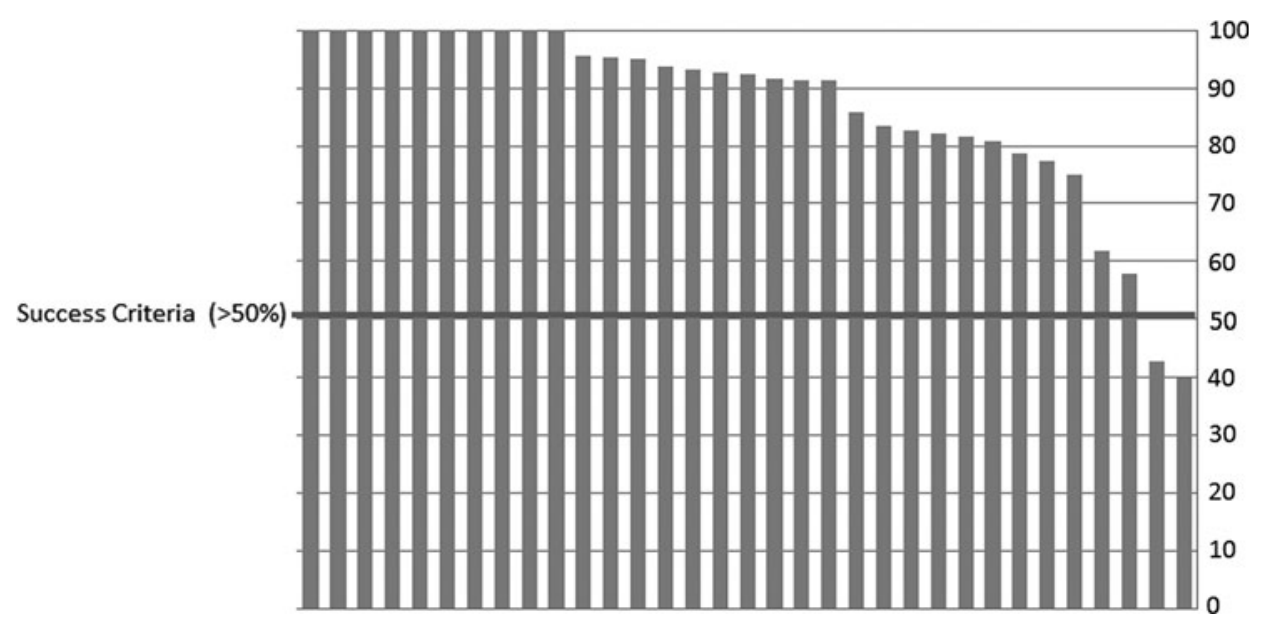

FIG. 1. Percentage reduction in total Gastroesophageal Reflux Disease-Health Related Quality of Life questionnaire score at Year 5. Each bar represents 1 of 33 patients who completed the questionnaire at 5 years. The data shown by each bar are the percentage reduction in total Gastroesophageal Reflux Disease-Health Related Quality of Life score at Year 5 compared with the total questionnaire score at baseline for each patient. A reduction of at least $50 \%$ was considered a success. The success criterion of Gastroesophageal Reflux Disease-Health Related Quality of Life score reduction was met by 93.9\% (31/ 33) of the patients.

standard deviation were used to describe continuous variables such as patient demographic data and baseline characteristics. Categorical demographic and baseline variables were summarized via frequency distributions, with the Wilcoxon sign rank test used for continuous outcomes and McNemar's test used for categorical normalization outcomes. Statistical significance was indicated by a $P$ value of $\leq .05$.

\section{Results}

In total, 44 patients (26 males and 18 females) were implanted with the MSAD between February 2007 and October 2008. The mean age of the study group was 42.8 (range, 1971) years, and the mean body mass index was 25.7 (range, $19.0-38.0) \mathrm{kg} / \mathrm{m}^{2}$. Thirty-three patients $(75 \%)$ were followed at 5 years. Noncompleters at Year 5 included voluntarily withdrawal $(n=2)$, study completion at Year 1 per protocol $(n=2)$, loss to follow-up $(n=4)$, and device removal $(n=3)$.

\section{GERD-HRQL scores, satisfaction, and side effects}

The mean total GERD-HRQL score off PPIs decreased from 25.7 at baseline to 2.9 at Year $5(P<.001)$, and $93.9 \%$ $(31 / 33)$ of patients had a greater than $50 \%$ reduction in total score compared with baseline (Fig. 1). A significant improvement in GERD-HRQL score after MSAD placement was reflected by a high level of patient satisfaction, with $90.9 \%$ of patients reporting being satisfied with their current condition at 5 years compared with no patients $(0 \%)$ reporting being satisfied at baseline. Side effects typically associated with antireflux surgery, such as difficulty swallowing and gas bloat, were no more bothersome or improved after MSAD placement compared with baseline (Table 1).

\section{Esophageal $\mathrm{pH}$ measurements}

Esophageal $\mathrm{pH}$ testing off PPI therapy demonstrated each patient had pathologic esophageal acid exposure at baseline. The mean percentage of time that $\mathrm{pH}$ was $<4$ decreased from
$11.9 \%$ at baseline to $4.6 \%$ at 5 years $(P<.001)$, and all other DeMeester components were reduced at 5 years compared with baseline (Table 2). Eighty-five percent (17/20) of patients who completed esophageal $\mathrm{pH}$ monitoring at 5 years achieved either normal esophageal acid exposure or had at

Table 1. Summary of Gastroesophageal Reflux Disease-Health Related Quality of Life ScORES By Question

\begin{tabular}{|c|c|c|}
\hline & $\begin{array}{c}\text { Baseline } \\
(\mathrm{n}=44)\end{array}$ & $\begin{array}{l}\text { Year } 5 \\
(\mathrm{n}=33)\end{array}$ \\
\hline How bad is your heartburn? & $3.7(4.0)$ & $0.5(0)$ \\
\hline Heartburn when lying down? & $3.1(3.0)$ & $0.5(0)$ \\
\hline Heartburn when standing up? & $3.3(3.0)$ & $0.4(0)$ \\
\hline Heartburn after meals? & $3.6(4.0)$ & $0.8(1)$ \\
\hline Does heartburn change your diet? & $3.1(4.0)$ & $0.2(0)$ \\
\hline $\begin{array}{l}\text { Does heartburn wake you } \\
\text { from sleep? }\end{array}$ & $2.5(3.0)$ & $0.1(0)$ \\
\hline $\begin{array}{l}\text { Do you have difficulty } \\
\text { swallowing? }\end{array}$ & $1.2(1.0)$ & $0.2(0)$ \\
\hline $\begin{array}{l}\text { Do you have bloating and } \\
\text { gassy feelings? }\end{array}$ & $2.9(3.0)$ & $0.3(0)$ \\
\hline $\begin{array}{l}\text { Do you have pain with } \\
\text { swallowing? }\end{array}$ & $0.6(0.0)$ & $0.0(0)$ \\
\hline $\begin{array}{l}\text { If you take medication, } \\
\text { does this affect your daily life? }\end{array}$ & $2.0(2.0)$ & $0.0(0)$ \\
\hline Total GERD-HRQL score & $25.7 \pm 6.4$ & $2.9 \pm 3.0$ \\
\hline
\end{tabular}

Values are mean (median) values except for total gastroesophageal reflux disease Health Related Quality of Life (GERD-HRQL) score, which is mean \pm standard deviation. The questionnaire was completed while the patient was off proton pump inhibitors for at least 10 days at both time points. For each question, patients were asked to rate their response on a scale of 0 to 5 (where $0=$ no symptoms, $1=$ symptoms noticeable but not bothersome, $2=$ symptoms noticeable and bothersome but not every day, $3=$ symptoms bothersome every day, $4=$ symptoms affect daily activities, and $5=$ symptoms are incapacitating, unable to do activities). The responses to each question were totaled to provide a total GERDHRQL score. Total scores range from 0 to 50 , with higher scores indicating worse symptoms. 
Table 2. Esophageal pH Measurements

\begin{tabular}{lccr}
\hline Measure & $\begin{array}{c}\text { Baseline } \\
(\mathrm{n}=44)\end{array}$ & $\begin{array}{c}\text { Year 5 } \\
(\mathrm{n}=20)\end{array}$ & $\begin{array}{c}\mathrm{P} \\
\text { value }\end{array}$ \\
\hline Total time (\%) & & & \\
pH <4 & 11.9 & 4.6 & $<.001$ \\
$\quad$ Upright & 13.6 & 5.6 & $<.001$ \\
$\quad \begin{array}{l}\text { Supine } \\
\text { Reflux episodes }\end{array}$ & 8.3 & 1.9 & .038 \\
$\quad$ Total number & 112.5 & 78.0 & .393 \\
$\quad$ Number lasting & 7.0 & 4.4 & .081 \\
$\quad$ >5 minutes & & & \\
$\quad$ Longest (minutes) & 37.4 & 19.3 & .015 \\
DeMeester score & 42.3 & 16.1 & $<.001$ \\
\hline
\end{tabular}

All testing was performed with the patient off proton pump inhibitors for at least 10 days.

least a $50 \%$ reduction from baseline. Normalization of esophageal $\mathrm{pH}$ (total percentage of time $<5.3 \%$ ) was achieved by $70 \%(14 / 20)$ of patients evaluated at 5 years.

\section{PPI use}

All patients were taking daily PPIs (single or double dose) to manage their primary symptom of heartburn at baseline. Complete cessation of PPIs at 5 years was achieved by $87.8 \%$ $(29 / 33)$ of patients, and a reduction of $50 \%$ or more in the average daily dose of PPIs was demonstrated by $93.9 \%$ (31/ 33) of patients (Table 3 ).

\section{Long-term safety}

Adverse events were assessed from the time of implant through the final 5-year visit. There were no reports of death, device erosions, device migrations, device malfunctions, or late-occurring device complications. No new safety risks were identified related to the implant procedure or device. During the entire course of the 5-year study, the rate of serious adverse events (SAEs) related to the device and/or implant procedure was $6.8 \%$ (3/44). All SAEs occurred and resolved within the first year after implant, with no related SAEs occurring after the first postoperative year. One patient experienced chest pain shortly after the implant requiring a short stay in the hospital, and the pain resolved less than 1 month after onset. Another patient experienced vomiting and nausea immediately following the implant procedure, which resulted in an extended hospital stay, with resolution of symptoms 5 days after the implant procedure. A third patient

Table 3. Summary of Key Clinical Outcomes

\begin{tabular}{lc} 
Clinical outcome & $\begin{array}{c}\text { Patients achieving } \\
\text { at Year 5 }\end{array}$ \\
\hline Improved GERD-HRQL $^{\mathrm{a}}$ & $93.9 \%(31 / 33)$ \\
Discontinuation of PPIs $^{\mathrm{b}}$ & $87.8 \%(29 / 33)$ \\
Reduced esophageal acid $^{\mathrm{b}}$ & $85.0 \%(17 / 20)$ \\
Satisfaction & $90.9 \%(30 / 33)$
\end{tabular}

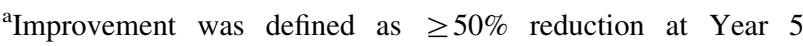
compared with baseline for total Gastroesophageal Reflux DiseaseHealth Related Quality of Life (GERD-HRQL) score.

${ }^{b}$ Normalization or $\geq 50 \%$ reduction of total acid exposure time at Year 5 compared with baseline by esophageal $\mathrm{pH}$ monitoring.

PPI, proton pump inhibitor.
}

had persistent dysphagia, which led to device removal 226 days postimplant. The patient was converted to a Nissen fundoplication and reported resolution of dysphagia approximately 30 days after device removal.

In addition to this device removal, two other patients had the device electively removed for reasons unrelated to an adverse event. One patient had the device explanted 468 days after implant to undergo magnetic resonance imaging (MRI), and the other patient had the device explanted at 1302 days to have a Nissen fundoplication for ongoing reflux symptoms.

All device removals were performed safely by a laparoscopic procedure with no complications.

\section{Discussion}

This 5-year study of magnetic sphincter augmentation confirms durable and sustained clinical outcomes out to 5 years. All reported studies with long-term clinical outcomes are in agreement that magnetic sphincter augmentation provides a sustained and high rate of symptom relief, discontinuation of PPIs, minimum side effects, and long-term safety. This report is now the second 5-year report and corroborates the findings of a larger 5-year study. ${ }^{14}$ The 44 implant procedures performed in this study were the first in the world. Today the authors have a combined experience that exceeds 350 implant procedures. Patient selection in this study was similar to the pivotal and other studies reported to date, which have largely enrolled patients with abnormal esophageal $\mathrm{pH}$, persistent symptoms despite PPIs, no or small hernia, no Barrett's esophagus, normal motility, and a body mass index of $<35 \mathrm{~kg} / \mathrm{m}^{2}$. When MSAD was used in these patients, results have been consistent and reproducible across multiple studies. The role of MSAD in patients beyond these parameters is likely to become clearer as its use expands in clinical practice.

The learning curve for placement of the MSAD was not steep, and we expect the same to be true for other surgeons who have experience with Nissen fundoplication and/or gastric banding. Mastering implantation of MSAD requires a focus on minimizing the amount of dissection performed and carefully locating and dissecting the posterior vagus nerve from the esophageal wall. The minimal dissection approach for MSAD deviates from the extensive dissection typical of a Nissen fundoplication. As a result, when a surgeon is first learning the procedure, placement of the MSAD requires discipline in order to avoid reverting back to the dissection technique used to create a fundus wrap.

Although the MSAD was not directly compared with Nissen fundoplication in this study, data from other clinical studies have provided evidence that side effects typically associated with Nissen fundoplication are less frequent and severe after LINX. ${ }^{15-17}$ Reynolds et al. ${ }^{15}$ found that severe gas bloat occurred in none of the patients after LINX but did occur in $10.6 \%$ of patients after Nissen fundoplication $(P=.022)$. Higher rates of gas bloat after fundoplication were also found in a large multicenter study where bothersome gas bloat occurring at least daily was $10 \%$ after LINX and $32 \%$ after fundoplication $(P<.001) .^{16}$

It is important that no new safety risks were identified during the 5-year follow-up. Overall, no device erosions or migrations have been reported during the studies with 5-year follow-up, providing reasonable assurance that the rate of 
device erosion and migration does not appear to increase with longer implant durations. A low incidence rate has been reported in other studies, but this rate remains less than $0.5 \%$ and appears to mostly occur within 2 years of the implant procedure. ${ }^{18}$ When device erosions have occurred, the events were managed on a nonemergency basis without further complication. ${ }^{18,19}$ The risk of device migration has been minimized by identifying and preserving the phrenoesophageal ligament combined with creation of a tunnel between the posterior wall of the esophagus and the posterior vagus nerve, providing a small space that limits movement of the device once implanted. ${ }^{14}$ To date, no reports of device migration have been reported.

The re-operation rate for LINX at 5 years was favorable and lower than rates reported after Nissen fundoplication for a comparable follow-up period: the re-operation rate was $6.8 \%$ at 5 years after LINX compared with $13.1 \%$ and $15.2 \%$ after Nissen fundoplication at 5 years. ${ }^{20,21}$ These re-operation rates, for both procedures, reflect early clinical experience, and later studies have reported lower re-operation rates for both. ${ }^{18,22}$ A comparison of re-operation rates is difficult to discern from the literature between MSAD and Nissen fundoplication; in general, the rates are acceptable for both procedures. The reasons for device removal in this study were similar to those in other reports and included dysphagia, continued reflux symptoms, and planned MRI imaging. Device explant for dysphagia typically occurs within 6 months of the implant procedure, but there has been a report of a late removal for postoperative dysphagia that persisted until the patient elected to have the device removed prior to exiting the study at about 5 years after implantation. ${ }^{14}$

In comparison with revision of a Nissen fundoplication, explant of MSAD is relatively straightforward, is associated with less risk, and leaves open the option for other treatments, including Nissen fundoplication. Re-operation following Nissen fundoplication is technically challenging and associated with a higher rate of complications and morbidity than the primary surgery. ${ }^{23,24}$ Scarring and herniation of the fundus into the chest can severely distort the gastric anatomy, and the stomach may not be suitable for esophageal reconstruction. ${ }^{25,26}$ The ability to safely remove the device, while leaving native gastric anatomy intact, should be considered an important advantage of the MSAD.

Areas of interest with MSAD include large hernias, sleeve gasterectomy, and post-radiofrequency ablation for Barrett's esophagus. In regard to hernias, the use of MSAD is not contraindicated per the approved regulatory labeling. However, clinical studies demonstrating the safety and efficacy of MSAD in larger hernias $(>3 \mathrm{~cm})$ have not been reported. It is the authors' opinion that magnetic sphincter augmentation is feasible in larger hernias based on our limited clinical experience with the MSAD in these patients, and therefore a $>3-\mathrm{cm}$ hernia should not preclude implantation of the device. In cases with larger hernias, the implant procedure would involve dissection of the lower mediastinum, similar to that routinely performed in conjunction with a Toupet or Nissen fundoplication, along with crural reapproximation. Pilot studies are currently underway to evaluate MSAD in patients with larger hernias. For reflux after sleeve gastrectomy, MSAD is well suited for these patients because a gastric fundus is no longer available for a Nissen fundoplication. The use of MSAD after sleeve gastrectomy has been reported in a small number of patients with favorable results. ${ }^{27}$ Clinical ex- perience with MSAD after radiofrequency ablation for Barrett's esophagus is not currently available in the literature but is feasible based on our limited experience. Patients should be alerted about the need for continued endoscopic surveillance.

When introduced, patients were instructed not to have MRI scans after MSAD implant. The model of device used in this study has since received conditional MRI approval for machines using up to $0.7 \mathrm{~T}$. More recently, a newer model of MSAD has been introduced for use in MRI machines up to 1.5 $\mathrm{T}$, providing patients with more options for diagnostic imaging. Patients with suspected or known allergies to titanium, stainless steel, nickel, or ferrous materials continue to be contraindicated for MSAD.

Limitations of this study included no comparison treatment group and loss of patients during the 5-year follow-up. Esophageal $\mathrm{pH}$ data were completed by all sites at 1 year, but not all sites continued to perform esophageal $\mathrm{pH}$ monitoring past the 1-year follow-up. Long-term manometric data to characterize any changes in esophageal motility would have been informative but were not part of the protocol past the 1year follow-up. No significant findings were found during follow-up to make continued manometric evaluations clinically necessary. For the clinical study, only patients with normal motility were eligible for MSAD implantation, defined as esophageal amplitude of at least $35 \mathrm{~mm} \mathrm{Hg}$ and at least $70 \%$ effective swallows. In clinical practice, we have adhered to implanting only patients with normal motility. Normal esophageal motility is necessary to facilitate propulsion of a food bolus through the esophageal body in order to push open the magnetic device and allow passage through the LES and into the stomach. In clinical practice, one author (J.C.L.) has developed a barium esophagram protocol to evaluate for adequate motility related to MSAD and limits the use of manometry to those patients found to have abnormal passage of a solid bolus by video esophagram. The protocol involves evaluations in the upright, prone, and supine positions while the patient swallows liquid as well as solid food bolus (i.e., contrast-coated hamburger).

\section{Conclusions}

The magnetic sphincter augmentation procedure is a major advancement in antireflux surgery because it enables restoration of the antireflux barrier without extensive anatomical disruption. No significant or new safety issues were identified during long-term follow-up. Safety was matched by durable clinical benefits, such as objective evidence of reduced acid exposure along with symptom improvement, increased patient satisfaction, and elimination of PPI use. When offered as a first-line surgical option, MSAD uses minimal dissection to restore the antireflux barrier and preserves the gastric anatomy. This study shows the relative safety and efficacy of magnetic sphincter augmentation for GERD.

\section{Acknowledgments}

This work was funded by Torax Medical.

\section{Disclosure Statement}

L.B. and J.C.L. have received payment from Torax Medical for consulting. G.S., D.D., and R.A.G. declare no competing interests exist. 


\section{References}

1. Vakil N, van Zanten SV, Kahrilas P, Dent J, Jones R, et al. The Montreal definition and classification of gastroesophageal reflux disease: A global evidence-based consensus. Am J Gastroenterol 2006;101:1900-1920.

2. Ferraro P, Duranceau A. Medical management of gastroesophageal reflux disease. Chest Surg Clin N Am 2001;11: 517-522.

3. Kahrilas PJ, Jonsson A, Denison H, Wernersson B, Hughes $\mathrm{N}$, Howden CW. Regurgitation is less responsive to acid suppression than heartburn in patients with gastroesophageal reflux disease. Clin Gastroenterol Hepatol 2012;10: 612-619.

4. Blonski W, Vela MF, Castell DO. Comparison of reflux frequency during prolonged multichannel intraluminal impedance and $\mathrm{pH}$ monitoring on and off acid suppression therapy. J Clin Gastroenterol 2009;43:816-820.

5. Vela MF, Camacho-Lobato L, Srinivasan R, Tutuian R, Katz PO, Castell DO. Simultaneous intraesophageal impedance and $\mathrm{pH}$ measurement of acid and nonacid gastroesophageal reflux: Effect of omeprazole. Gastroenterology 2001;120:1599-1606.

6. Catarci M, Gentileschi P, Papi C, Carrara A, Marrese R, Gaspari AL, Grassi GB. Evidence-based appraisal of antireflux fundoplication. Ann Surg 2004;239:325-337.

7. Ferguson CM, Rattner DW. Initial experience with laparoscopic Nissen fundoplication. Am Surg1995;61:21-23.

8. Tosato F, Marano S, Luongo B, Paltrinieri G, Portale G, Mattacchione S, Bezzi M. Partial or total fundoplication (with or without division of the short gastric vessels): Which is the best laparoscopic choice in GERD surgical treatment? Surg Laparosc Endosc Percutan Tech 2010;20: 371-377.

9. Ganz RA, Gostout CJ, Grudem J, Swanson W, Berg T, DeMeester TR. Use of a magnetic sphincter for the treatment of GERD: A feasibility study. Gastrointest Endosc 2008;67:287-294.

10. Bonavina L, Saino GI, Bona D, Lipham J, Ganz RA, Dunn $\mathrm{D}$, DeMeester T. Magnetic augmentation of the lower esophageal sphincter: Results of a feasibility clinical trial. J Gastrointest Surg 2008;12:2133-2140.

11. Bonavina L, DeMeester T, Fockens P, Dunn D, Saino G, Bona D, Lipham J, Bemelman W, Ganz RA. Laparoscopic sphincter augmentation device eliminates reflux symptoms and normalizes esophageal acid exposure: One- and 2-year results of a feasibility trial. Ann Surg 2010;252:857-862.

12. Lipham JC, DeMeester TR, Ganz RA, Bonavina L, Saino G, Dunn DH, Fockens P, Bemelman W. The LINX ${ }^{\circledR}$ reflux management system: Confirmed safety and efficacy now at 4 years. Surg Endosc 2012;26:2944-2949.

13. Velanovich V. Comparison of generic (SF-36) vs. disease specific quality-of-life (GERD-HRQL) scales for gastroesophageal disease. J Gastrointest Surg 1998;2:141-145.

14. Ganz RA, Edmundowicz SA, Taiganides PA, Lipham JC, Smith CD, DeVault KR, et al. Long-term outcomes of patients receiving a magnetic sphincter augmentation device for gastroesophageal reflux. Clin Gastroenterol Hepatol 2015 June 2 [Epub ahead of print]. doi: 10.1016/j.cgh .2015.05.028.

15. Reynolds JL, Zehetner J, Wu P, Shah S, Bildzukewicz N, Lipham JC. Laparoscopic magnetic sphincter augmentation vs laparoscopic Nissen fundoplication: A matched- pair analysis of 100 patients. J Am Coll Surg 2015;221: 123-128.

16. Riegler M, Schoppman SF, Bonavina L, Ashton D, Horbach T, Kemem M. Magnetic sphincter augmentation and fundoplication for GERD in clinical practice: One-year results of a multicenter, prospective observational study. Surg Endosc 2015;29:1123-1129.

17. Louie BE, Farivar AS, Schultz D, Brennan C, Valliéres E, Aye RW. Short-term outcomes using magnetic sphincter augmentation versus Nissen fundoplication for medically resistant gastroesophageal reflux disease. Ann Thorac Surg 2014;98:498-505.

18. Lipham JC, Taiganides PA, Louie BE, Ganz RA, DeMeester TR. Safety analysis of first 1000 patients treated with magnetic sphincter augmentation for gastroesophageal reflux disease. Dis Esophagus 2015;28:305-311.

19. Bauer M, Meining A, Kranzfelder M, et al. Endoluminal perforation of a magnetic antireflux device. Surg Endosc 2015 April 16 [Epub ahead of print]. doi: 10.1007/s00464015-4145-6.

20. Draaisma WA, Rijnhart-de Jong HG, Broeders IA, Smout AJ, Furnee EJ, Gooszen HG. Five-year subjective and objective results of laparoscopic and conventional Nissen fundoplication: A randomized trial. Ann Surg 2006;244: 33-41.

21. Lafullarde T, Watson DI, Jamieson GG, Myers JC, Game PA, Devitt PG. Laparoscopic Nissen fundoplication: Fiveyear results and beyond. Arch Surg 2001;136:180-184.

22. Zhou T, Harnsberger C, Broderick R, Fuchs H, Talamini M, Jacobsen G, Horgan S, Chang D, Sandler B. Reoperation rates after laparoscopic fundoplication. Surg Endosc 2015;29:510-514.

23. Furnee EJ, Draaisma WA, Broeders IA, Smout AJ, Gooszen HG. Surgical reintervention after antireflux surgery for gastroesophageal reflux disease: A prospective cohort study in 130 patients. Arch Surg 2008;143:267-274.

24. Furnée EJ, Draaisma WA, Broeders IA, Gooszen HG. Surgical reintervention after failed antireflux surgery: A systematic review of the literature. J Gastrointest Surg 2009;13:1539-1549.

25. Symons NR, Purkayastha S, Dillemans B, Athanasiou T, Hanna GB, Darzi A, Zacharakis E. Laparoscopic revision of failed antireflux surgery: A systematic review. Am J Surg 2011;202:336-343.

26. Shen KR, Harrison-Phipps KM, Cassivi SD, Wigle D, Nichols FC 3rd, Allen MS, Wood CM, Deschamps C. Esophagectomy after anti-reflux surgery. J Thorac Cardiovasc Surg 2010;139:969-975.

27. Desart K, Rossidis G, Michel M, Lux T, Ben-David K. Gastroesophageal reflux management with the LINX $^{\circledR}$ system for gastroesophageal reflux disease following laparoscopic sleeve gastrectomy. J Gastrointest Surg 2015 July 11 [Epub ahead of print]. doi: 10.1007/s11605-015-2887-z.

Address correspondence to: Robert A. Ganz, MD Minnesota Gastroenterology, PA 15700 37th Avenue North, Suite \#300 Plymouth, MN 55446

E-mail: gastrodude@visi.com 